\title{
Validating precision estimates in horizontal wind measurements from a Doppler lidar
}

\author{
Rob K. Newsom ${ }^{1}$, W. Alan Brewer ${ }^{2}$, James M. Wilczak ${ }^{2}$, Daniel E. Wolfe ${ }^{2,3}$, Steven P. Oncley ${ }^{4}$, and \\ Julie K. Lundquist ${ }^{5,6}$ \\ ${ }^{1}$ Pacific Northwest National Laboratory, Richland, WA 99352, USA \\ ${ }^{2}$ National Oceanic and Atmospheric Administration, Earth System Research Laboratory, Boulder, CO 80305, USA \\ ${ }^{3}$ Cooperative Institute for Research in Environmental Sciences, Boulder, CO 80305, USA \\ ${ }^{4}$ National Center for Atmospheric Research, Boulder, CO 80307, USA \\ ${ }^{5}$ Department of Atmospheric and Oceanic Sciences, University of Colorado, Boulder, CO 80309, USA \\ ${ }^{6}$ National Renewable Energy Laboratory, Golden, CO 80401, USA \\ Correspondence to: Rob K. Newsom (rob.newsom@pnnl.gov)
}

Received: 23 September 2016 - Discussion started: 22 November 2016

Revised: 3 February 2017 - Accepted: 27 February 2017 - Published: 30 March 2017

\begin{abstract}
Results from a recent field campaign are used to assess the accuracy of wind speed and direction precision estimates produced by a Doppler lidar wind retrieval algorithm. The algorithm, which is based on the traditional velocityazimuth-display (VAD) technique, estimates the wind speed and direction measurement precision using standard error propagation techniques, assuming the input data (i.e., radial velocities) to be contaminated by random, zero-mean, errors. For this study, the lidar was configured to execute an 8-beam plan-position-indicator (PPI) scan once every 12 min during the 6-week deployment period. Several wind retrieval trials were conducted using different schemes for estimating the precision in the radial velocity measurements. The resulting wind speed and direction precision estimates were compared to differences in wind speed and direction between the VAD algorithm and sonic anemometer measurements taken on a nearby $300 \mathrm{~m}$ tower.

All trials produced qualitatively similar wind fields with negligible bias but substantially different wind speed and direction precision fields. The most accurate wind speed and direction precisions were obtained when the radial velocity precision was determined by direct calculation of radial velocity standard deviation along each pointing direction and range gate of the PPI scan. By contrast, when the instrumental measurement precision is assumed to be the only contribution to the radial velocity precision, the retrievals resulted
\end{abstract}

in wind speed and direction precisions that were biased far too low and were poor indicators of data quality.

\section{Introduction}

Coherent Doppler lidars (CDL) are used in applications ranging from basic atmospheric boundary-layer research to model data assimilation (Riishøjgaard et al., 2004; Chai et al., 2004; Newsom and Banta, 2004a, b; Newsom et al., 2005; Weissmann and Cardinali, 2007; Pu et al., 2010) and to wind resource assessment (Pena et al., 2009; Lang and McKeogh, 2011; Koch et al., 2012; Pichugina et al., 2012; Hsuan et al., 2014; Newsom et al., 2015; Newman et al., 2016; Choukulkar et al., 2016). Within the wind energy industry, these instruments are viewed as cost-effective alternatives to instrumented towers for wind resource assessment provided the measurement uncertainties are within acceptable limits.

Quantifying the uncertainty in CDL-derived wind speed and direction measurements is perhaps best achieved by comparison with collocated in situ reference measurements. For wind resource assessment, the International Energy Agency's recommended best practices (Clifton et al., 2013) state that CDLs should undergo periodic verification using anemometers that have been calibrated against a traceable standard. This enables determination of uncertainty due to both the random error (i.e., precision) and systematic error 

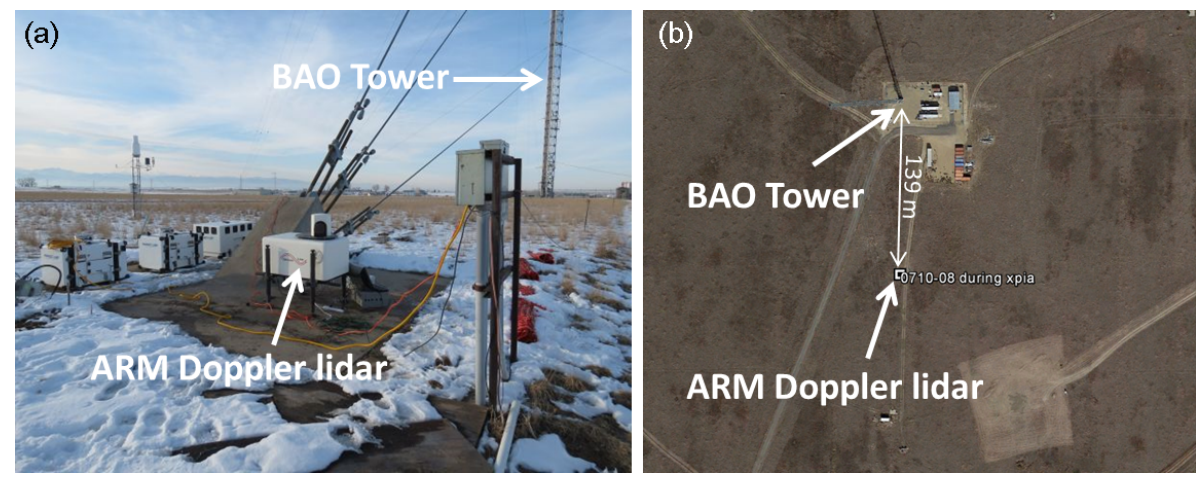

Figure 1. (a) Setup of the ARM Doppler lidar and (b) aerial view showing the location of the lidar relative to the BAO tower during XPIA.

(i.e., bias). Although these verification studies are valuable, one problem is that they are invariably carried out at locations different from the resource assessment site. Thus, the uncertainties determined in this manner may or may not be applicable to the measurements obtained at the resource assessment site. It is, however, possible to obtain "on-site" estimates of the wind speed and wind direction precision using standard error propagation techniques in the CDL wind retrieval algorithm if the variability in the radial velocity measurements can be properly characterized. These precision estimates could then be used as measures of data quality.

A number of studies have been performed to characterize uncertainties in CDL wind retrievals (Gottschall et al., 2012; Lane et al., 2013; Davies et al., 2003). Lundquist et al. (2015) examined errors caused by inhomogeneous flow using a lidar simulator approach in which high-resolution LES output was used as the reference field. Wang et al. (2015) used field measurements to investigate uncertainties in winds retrieved from sector scans and found the radial velocity variance to be a robust measure of the uncertainty in the retrieved winds due to its relationship with turbulence.

In this study, we evaluate the accuracy of CDL-derived wind speed and wind direction precision estimates obtained by propagating radial velocity uncertainties through a velocity-azimuth-display (VAD) algorithm. The VAD algorithm uses data from a plan-position-indicator (PPI) scan to generate estimates of wind speed and direction as a function of height. PPI scans are performed by scanning the lidar beam in azimuth while maintaining a constant elevation angle. A key assumption in the algorithm is that the flow is horizontally homogeneous. For perfectly horizontally homogeneous flow in the absence of turbulence or measurement error, the radial velocity versus azimuth curve forms a perfect sinusoid. In reality, deviations from the perfect sinusoid occur due to spatial and temporal fluctuations in the velocity field and instrumental errors. In the context of the VAD algorithm, any departure from the perfect sinusoid may be regarded as error. If the random uncertainty (i.e., precision) of the radial velocity measurements are known, then it is possible to estimate the precision (i.e., random uncertainty) in the wind speed and direction using standard error propagation techniques.

In this study we are concerned with evaluating the representativeness of wind and direction precision estimates using different methods for estimating the precision (i.e., random uncertainty) of the input radial velocity data. This is accomplished using data that were acquired during the eXperimental Planetary boundary-layer Instrument Assessment (XPIA) field campaign (Lundquist et al., 2016) at the Boulder Atmospheric Observatory (BAO).

During XPIA, several CDL systems were deployed on or near the BAO site for the purpose of assessing the accuracy of single- and multiple-Doppler retrievals of 3-D wind fields. The $300 \mathrm{~m}$ BAO tower was instrumented with pairs of sonic anemometers on opposing booms at six levels, thus providing accurate reference measurements against which the CDL wind retrievals could be compared. In this study, we focus specifically on the wind speed and direction retrievals from a single CDL system.

The CDL used in this study was on loan from the US Department of Energy's Atmospheric Radiation Measurement (ARM) program (Mather and Voyles, 2014) and represents one of nine CDL systems that ARM currently operates at various sites around the world in support of climate research. The CDLs at the ARM sites spend the bulk of their time staring vertically acquiring high-temporal-resolution $(\sim 1 \mathrm{~Hz})$ measurements of vertical velocity. The vertical stares are momentarily interrupted once every 10 to $15 \mathrm{~min}$ to perform an 8-beam step-stare PPI scan, from which profiles of horizontal wind speed and direction are derived. This simple scanning strategy was designed to provide well-resolved measurements of the horizontal winds while minimizing gaps in the vertical velocity time series, which is essential for computing turbulent power spectra. During XPIA, the CDL used in this study was operated in a similar manner so that the results could be readily transferred to ARM operations.

This paper is organized as follows. Section 2 describes the experimental setup, including a brief description of the instrumentation on the $300 \mathrm{~m}$ BAO tower as well as the configuration and scan strategy used by the CDL. The lidar wind 
retrieval algorithm and error propagation methods are presented in Sect. 3. Section 4 presents the results of three wind retrieval trials using the sonic anemometers on the $300 \mathrm{~m}$ BAO tower as reference measurements.

\section{Experimental setup and instrumentation}

Prior to decommissioning in 2016, the BAO was a research facility maintained by NOAA's Earth System Research Laboratory (Kaimal and Gaynor, 1983). The facility was located on Colorado's High Plains approximately $25 \mathrm{~km}$ east of Boulder, Colorado $\left(40.05^{\circ} \mathrm{N}, 105.00^{\circ} \mathrm{W}\right)$. The center piece of the BAO site was a $300 \mathrm{~m}$ meteorological tower. Figure 1 shows the set up during XPIA and the location of the CDL relative to the $\mathrm{BAO}$ tower, $139 \mathrm{~m}$ south of the tower.

\subsection{Doppler lidar}

The CDL used in this study is a commercial-grade system manufactured by Halo Photonics. This particular system was first acquired by ARM in 2010 and was subsequently deployed to ARM's Tropical Western Pacific site in Darwin, Australia, before that site was decommissioned at the beginning of 2015. The system is currently one of five CDLs operating at ARM's Southern Great Plains site in north-central Oklahoma.

The CDL provides range-resolved measurements of attenuated aerosol backscatter, signal-to-noise ratio (SNR) and radial velocity. The system employs an eye-safe laser that transmits at a wavelength of $1.548 \mu \mathrm{m}$, with $\sim 150 \mathrm{~ns}$ $(22.5 \mathrm{~m})<100 \mu \mathrm{J}$ pulses at a rate of $15 \mathrm{kHz}$. The primary scattering mechanism is atmospheric aerosol. As a result, valid measurements are usually restricted to the atmospheric boundary layer where aerosol concentrations are high enough to ensure good SNR. The instrument incorporates a full upper hemispheric scanner that can be configured to operate in either step-stare or continuous scan mode. In stepstare mode, the CDL acquires data by dwelling with the beam in a fixed pointing direction and scans are performed by incrementally moving from one pointing direction to the next. In continuous scan mode, the CDL acquires data continuously as the scanner moves between predefined starting and ending positions. The internal processor can be configured to operate using a wide range of pulse accumulation times and range gate sizes. During XPIA, the system was operated using a range gate size of $30 \mathrm{~m}$ and 200 range gates, resulting in a maximum measurement range of about $6 \mathrm{~km}$. Typically, the maximum range for usable measurements varied between 1 and $3 \mathrm{~km}$, depending on atmospheric conditions. Further details about the ARM Doppler lidars can be found in Pearson et al. (2009) and Newsom (2012).

From 6 March to 16 April 2015 the ARM CDL was deployed within a small cluster of profiling CDLs approximately $140 \mathrm{~m}$ south of the BAO tower, as shown in Fig. 1 .
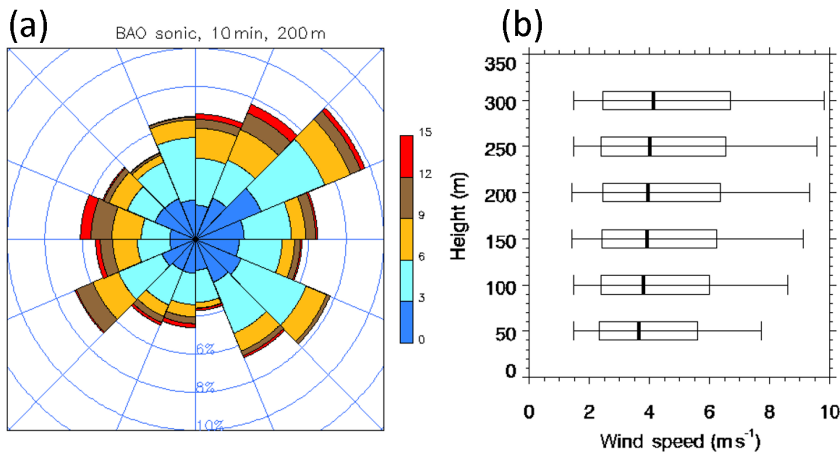

Figure 2. (a) Wind rose from $10 \mathrm{~min}$ averaged sonic anemometer data at the $200 \mathrm{~m}$ level on the BAO tower and (b) box and whisker plot of $10 \mathrm{~min}$ averaged wind speeds from the tower sonic anemometers. The box and whiskers indicate the 10th, 25th, 50th, 75th and 90th percentiles. Both plots use data spanning the period from 6 March through 16 April 2015.

During this time, the instrument was operated using a fixed scan schedule consisting of PPI scans once every $12 \mathrm{~min}$, 10 min tower stares once per hour and target sector scans once per day. The remainder (and majority) of the time was spent acquiring $1 \mathrm{~Hz}$ vertical velocity profiles.

PPI scans were performed using the step-stare scan mode at an elevation angle of $60^{\circ}$ and eight evenly spaced azimuth angles around the compass. The pulse integration time for each profile was set to $2 \mathrm{~s} \mathrm{(30000} \mathrm{laser} \mathrm{pulses),} \mathrm{and} \mathrm{the} \mathrm{time}$ required to execute one complete PPI scan was about $40 \mathrm{~s}$. We note that this is the same configuration that is currently used by most of the CDLs at the ARM sites.

The pointing accuracy of the lidar beams is crucial for determination of the wind direction. Although the angular precision of the scanner is about $\pm 0.05^{\circ}$, the pointing accuracy requires careful calibration. To ensure proper orientation relative to the vertical coordinate, the "levelness" of the instrument was routinely monitored throughout the deployment period, and target sector scans were performed on a daily basis in order to accurately determine the lidar's azimuthal pointing direction relative to true north. The target scan consisted of a high-angular-resolution narrow-sector continuousmode PPI scan in the general direction of a target of opportunity. The observed location of the hard target return in the scan data, together with the known GPS coordinates of the lidar and the target enabled us to determine of the lidar's orientation with respect to true north and to estimate any error in the reported range. In this case, no significant range errors were observed. For this experiment the target that was used was a tall stadium light post located next to the football field at Erie High School, at a distance of about $800 \mathrm{~m}$ west of the lidar location. 
(a)

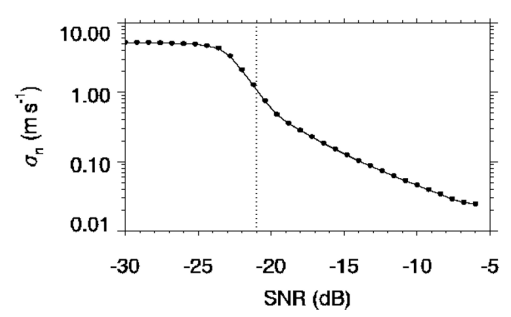

(b)

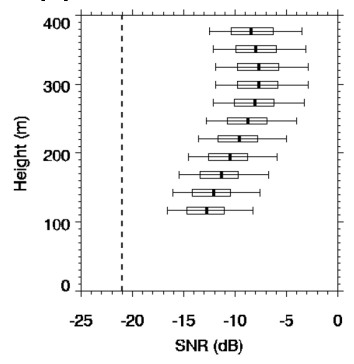

Figure 3. (a) The standard deviation of instrumental measurement precision, $\sigma_{\mathrm{n}}$, as a function of SNR, and (b) box and whisker plot of the SNR profile indicating the 10th, 25th, 50th, 75th and 90th percentiles. Both plots use data spanning the period from 6 March through 16 April 2015.

\subsection{BAO tower sonic anemometers}

During XPIA the BAO tower was instrumented at six levels $(50,100,150,200,250$ and $300 \mathrm{~m})$ with fast response $(20 \mathrm{~Hz})$ 3-D sonic anemometers (Campbell CSAT3). Each level had two sonic anemometers, one mounted on a southeast boom (at a heading angle of $154^{\circ}$ ) and one mounted on a northwest boom (at a heading angle of $334^{\circ}$ ). This improved the odds of obtaining measurements that were unaffected by the tower wake. Data from the sonic anemometers were tilt-corrected (Wilczak et al., 2001) and rotated into geographical coordinates, with positive $u$ toward the east, positive $v$ toward the north and $w$ pointing in the corrected vertical direction.

Figure 2 summarizes mean wind statistics as determined from $10 \mathrm{~min}$ average sonic anemometer data at the $200 \mathrm{~m}$ level on the BAO tower during the ARM CDL deployment period from 6 March through 16 April 2015. For each 10 min averaging interval we used the sonic anemometer on the upwind side of the tower, as tower wake effects were observed to be quite significant (McCaffrey et al., 2017). The wind rose shown in Fig. 2a indicates that the strongest winds tended to blow from the northeast and the west. Although there was no strongly preferred wind direction, there was a slightly higher occurrence of northeasterly flow during the deployment period. Winds were generally fairly light with the bulk of the wind speeds occurring between 3 and $6 \mathrm{~ms}^{-1}$. Figure $2 b$ indicates that the median wind speeds tended to be at or slightly below $4 \mathrm{~ms}^{-1}$. Inspection of the data shows that wind speeds exceeding $15 \mathrm{~ms}^{-1}$ occurred for brief periods on 16-17 March, 24-25 March, 12 April and 15-16 April. Overall, however, the winds rarely exceeded $10 \mathrm{~ms}^{-1}$ during the deployment period.

\section{Lidar wind retrieval and precision estimation}

CDL estimates of horizontal winds are computed from PPI scan data using a VAD algorithm based on the classic tech-

nique described by Browning and Wexler (1968). Assuming the flow to be horizontally uniform and steady at a given range gate or height above the ground, the wind velocity components are retrieved by fitting a sinusoid to the radial velocity data; the amplitude, phase and offset of the sinusoid determine the wind speed, wind direction and vertical velocity, respectively. This is equivalent to minimizing

$\psi^{2}=\sum_{i=1}^{N} \frac{\left(\boldsymbol{u}_{o} \boldsymbol{r}_{i}^{T}-u_{r i}\right)^{2}}{\sigma_{r i}^{2}}$

with respect to the components of the mean velocity vector, $\boldsymbol{u}_{o}=\left(u_{o}, v_{o}, w_{o}\right)$. In Eq. (1), $N$ is the number of beams in the PPI scan, $u_{r i}$ is a radial velocity measurement, $\sigma_{r i}$ is the measurement uncertainty due to random errors (i.e., precision), and $\boldsymbol{r}_{i}$ is a unit vector from the lidar to the measurement point, i.e., the beam pointing direction, and is given by

$\boldsymbol{r}_{i}=\left(\sin \phi_{i} \cos \theta, \cos \phi_{i} \cos \theta, \sin \theta\right)$,

where $\phi_{i}$ is the azimuth angle as measured clockwise from true north, $\theta$ is the (constant) elevation angle as measured from the horizontal plane and $\boldsymbol{r}_{i}^{T}$ is the transpose of $\boldsymbol{r}_{i}$. The summation in Eq. (1) is performed over all the pointing directions in the PPI scan. Minimizing Eq. (1) with respect to the components of $\boldsymbol{u}_{o}$ results in a system of three equations and three unknowns, the solution of which can be expressed as

$\boldsymbol{u}_{o}=\mathbf{C b}$,

where

$\mathbf{C}=\left(\sum_{i=1}^{N} \frac{\boldsymbol{r}_{i}^{T} \boldsymbol{r}_{i}}{\sigma_{r i}^{2}}\right)^{-1}$

is the covariance matrix, and

$\boldsymbol{b}=\sum_{i=1}^{N} \frac{u_{r i}}{\sigma_{r i}^{2}} \boldsymbol{r}_{i}^{T}$.

Equation (3) determines the wind velocity components at a fixed range gate. The height of the range gate above ground level is given by $z=r \sin \theta$, where $r$ is the distance from the lidar to the range gate center. Wind profiles are then constructed by applying Eq. (3) to all range gates.

When the individual radial velocity precisions, $\sigma_{r i}$, are known the precision of the retrieved velocity components can be obtained from the diagonal elements of the weighted covariance matrix (Press et al., 1988), i.e.,

$\sigma_{u}=\sqrt{C_{11}}$ and $\sigma_{v}=\sqrt{C_{22}}$.

When the radial velocity precisions are not known, the precisions in $u$ and $v$ can be estimated by setting $\sigma_{r i}=1$ in Eq. (1). 
Table 1. Radial velocity and retrieved parameter precision estimation schemes for trials 1,2 and 3 .

\begin{tabular}{lll}
\hline Trial & $\begin{array}{l}\text { Radial velocity } \\
\text { uncertainty }\end{array}$ & $\begin{array}{l}\text { Retrieved parameter } \\
\text { precision }\end{array}$ \\
\hline 1 & $\begin{array}{l}\sigma_{r i}=1 \\
\sigma_{r i} \text { is computed from nine } \\
\text { independent samples for } \\
\text { each pointing direction } \\
3\end{array}$ & $\begin{array}{l}\text { Estimated from Eq. (7) } \\
\text { Estimated from Eq. (6) }\end{array}$ \\
& $\sigma_{r i}=\sigma_{n i}$ & Estimated from Eq. (7) \\
\hline
\end{tabular}

The precisions of the retrieved velocity components are then determined in the following manner (Press et al., 1988):

$\sigma_{u}=\psi \sqrt{\frac{C_{11}}{N-N_{f}}}$ and, $\sigma_{v}=\psi \sqrt{\frac{C_{22}}{N-N_{f}}}$,

where $N_{f}$ is the number of retrieved parameters, i.e., $N_{f}=3$ for 3-D retrievals $\left(u_{o}, v_{o}\right.$ and $\left.w_{o}\right)$ or $N_{f}=2$ for 2-D retrievals $\left(u_{o}\right.$ and $v_{o}$ only).

Given the precisions for $u_{o}$ and $v_{o}$, the estimated precision in the retrieved wind speed is given by

$\sigma_{M}=\left(\left(u_{o} \sigma_{u}\right)^{2}+\left(v_{o} \sigma_{v}\right)^{2}\right)^{1 / 2} / M$,

where $M=\sqrt{u_{o}^{2}+v_{o}^{2}}$ is the CDL wind speed, and the estimated wind direction precision is given by

$\sigma_{\alpha}=\left(\left(u_{o} \sigma_{v}\right)^{2}+\left(v_{o} \sigma_{u}\right)^{2}\right)^{1 / 2} / M^{2}$

The key assumption underlying the retrieval method is that the mean winds are horizontally homogeneous over the time it takes to perform a PPI scan. The actual measured radial velocity contains contributions from turbulent velocity fluctuations, $\boldsymbol{u}^{\prime}$, and instrumental noise, $n$, such that

$u_{r}=\left(\boldsymbol{u}_{o}+\boldsymbol{u}^{\prime}\right) \boldsymbol{r}^{T}+n$

For any given pointing direction at a fixed range from the lidar we assume that the ensemble average of the turbulent and noise terms are zero and that the noise and turbulence are completely uncorrelated. In that case, the variance of the measured radial velocity can be written as

$\sigma_{\mathrm{r}}^{2}=\overline{u_{r}^{\prime 2}}+\sigma_{\mathrm{n}}^{2}$,

where $\overline{u_{r}^{\prime 2}}=\overline{\left(\boldsymbol{u}^{\prime} \bar{r}^{T}\right)^{2}}$ is the contribution from turbulence and $\sigma_{\mathrm{n}}$ is the standard deviation of the instrumental noise, which we refer to as the instrumental measurement precision.

\subsection{Instrumental measurement precision}

The instrumental measurement precision is defined as the random uncertainty in the radial velocity introduced by the
Doppler signal processor internal to the lidar. This uncertainty, which increases with decreasing SNR, is caused by random fluctuations in the Doppler shift estimates due primarily to frequency drift in the local oscillator (Frehlich, 2004). The performance of the CDL system can be partly characterized by parameterizing the instrumental measurement precision in terms of the SNR. This results in an empirical curve that is independent of range and atmospheric conditions.

Figure 3a shows the instrumental measurement precision as a function of the SNR for the ARM CDL. Precision estimates were obtained through analysis of the vertical staring data using the autocovariance technique described by Lenschow et al. (2000) and Pearson et al. (2009). For this study, the instrumental measurement precision and mean SNR were computed from $30 \mathrm{~min}$ time series of radial velocity. This analysis was performed using all contiguous $30 \mathrm{~min}$ time periods and range gates in the vertical staring data set collected during XPIA. This resulted in a large number of precision and mean SNR sample pairs. The result shown in Fig. 3a was obtained by fitting a smooth curve through these sample pairs.

All wind retrievals in this study were processed by requiring that the SNR be greater than or equal to 0.008 for each beam in a given PPI scan. This threshold level, which is indicated by the dotted and dashed vertical lines in Fig. 3, corresponds to an instrumental precision value of roughly $1 \mathrm{~ms}^{-1}$. As a result, radial velocities with $\sigma_{\mathrm{n}}$ greater than approximately $1 \mathrm{~ms}^{-1}$ were rejected and thus not used to compute the winds. We note that the SNR over the height of the BAO tower was almost entirely above threshold during the deployment period, as illustrated by the median SNR profile in Fig. 3b.

\section{Results}

\subsection{Precision estimation methods}

Three trials were conducted to evaluate different methods for estimating the radial velocity precision and the resultant precision in the derived wind components. For all of these trials, winds were computed from the $40 \mathrm{~s}$ PPI scans and the wind retrieval algorithm was configured to retrieve all three components of the wind field and use all eight beams of the PPI scans. All three trials use the same PPI scan data. The only difference between the trials is in the treatment of the radial velocity precision, $\sigma_{\mathrm{r}}$. These trials are described in Table 1.

Trial 1 uses the simplest method for estimating the precision and is currently the method used to process PPI scan data from all the existing ARM CDLs. We note that this approach is equivalent to assuming that the radial velocity precision is independent of the azimuth, i.e., isotropic, and thus independent of the mean wind direction. 

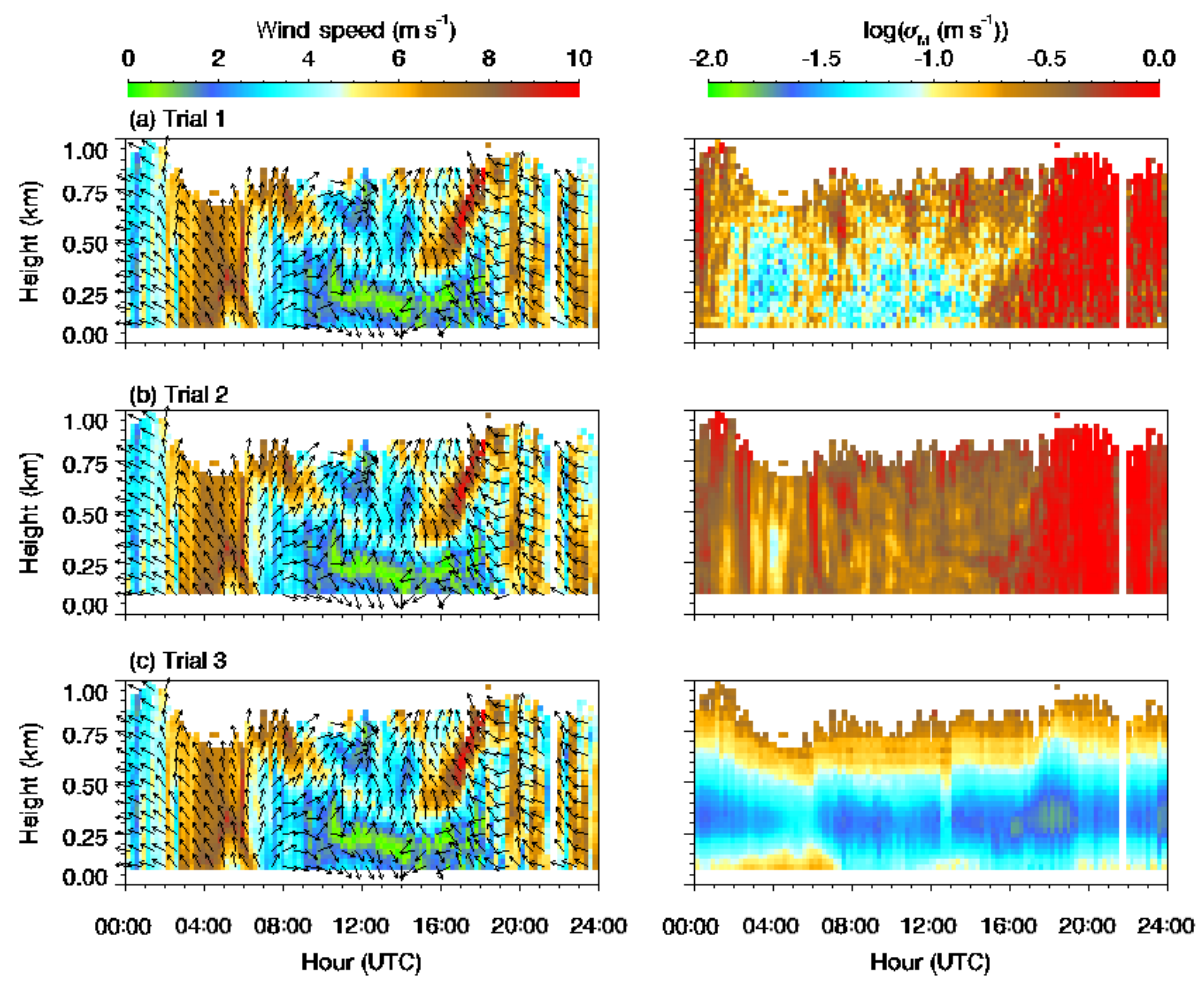

Figure 4. Time-height plots of the retrieved wind speed (left) and estimated wind speed precision (right) for 4 April 2015 for (a) Trial 1 , (b) Trial 2 and (c) Trial 3.

For Trial 2 radial velocity precision for a given pointing direction and range gate is estimated by computing the variance of the radial velocity over three consecutive scans and two neighboring range gates. Thus, for the $i$ th azimuth, $j$ th range gate and $q$ th PPI scan the radial velocity precision for Trial 2 is estimated from

$\sigma_{\mathrm{r}}^{q}\left(\phi_{i}, r_{j}\right)=\left[\frac{1}{9} \sum_{l=q-1}^{q+1} \sum_{k=j-1}^{j+1}\left(u_{r}^{l}\left(\phi_{i}, r_{k}\right)-\bar{u}_{r}^{q}\left(\phi_{i}, r_{j}\right)\right)^{2}\right]^{1 / 2}$,

where

$\bar{u}_{r}^{q}\left(\phi_{i}, r_{j}\right)=\frac{1}{9} \sum_{l=q-1}^{q+1} \sum_{k=j-1}^{j+1} u_{r}^{l}\left(\phi_{i}, r_{k}\right)$

The idea behind this scheme was to approximate a scanning strategy in which several independent radial velocity samples are recorded for each pointing direction and range gate. This provides a means of estimating the precision directly from the observations and avoids the assumption of isotropy inherent in Trial 1. In the present case, the PPI scans are spaced 12 min apart, so we assume the atmosphere to be statistically stationary over a 24 min period. We emphasize that the averaging scheme described above is only used to estimate the radial velocity precision. The radial velocity measurements used in Trial 2 are the same as those used in the other trials.

For Trial 3 the radial velocity uncertainty, $\sigma_{\mathrm{r}}$, is taken to be equal to the instrumental measurement precision, $\sigma_{\mathrm{n}}$, so that the effects of turbulence are completely ignored. Since the instrumental precision is parameterized in terms of the SNR (see Fig. 4a), the radial velocity uncertainty for Trial 3 is determined solely by the SNR.

Figure 4 shows representative lidar wind retrievals for 4 April 2015, with results for trials 1, 2 and 3 shown in panels (a), (b) and (c), respectively. The wind speed and direction retrievals (left) for all three trials look qualitatively similar, but the precision estimates (right) are substantially different, particularly between Trial 3 and either Trial 1 or 2 . The precision for trials 1 and 2 shows a strong diurnal dependence with larger random uncertainties during the daytime period (sunrise and sunset are about 12:00 and 02:00 UTC, respectively). The precision estimates for Trial 2 are larger than Trial 1 and exhibit more smoothing as a result of the averaging described by Eq. (12). By contrast, the precision estimates for Trial 3 are much smaller than either trials 1 or 2 and exhibit a completely different structure with no distinctive diurnal variation. The reason for this difference is that the radial velocity precisions for Trial 3 were determined solely from the SNR (see Fig. 3a). The SNR responds to variations in aerosol properties (including size distribution and number density) and is less affected by variations in atmospheric stability.

To enable comparison with the CDL-derived winds, $20 \mathrm{~Hz}$ data from each sonic anemometer on the BAO tower were 
Table 2. Results of the comparison between the lidar-derived winds and the sonic anemometers on the BAO tower. The results with 0 and $50 \%$ data rejection are shown. The averaging times for the sonic anemometer data were twice the PPI scan duration, or nominally about $80 \mathrm{~s}$.

\begin{tabular}{lrrr|rrr}
\hline Data rejection & \multicolumn{3}{c}{$0 \%$} & \multicolumn{3}{c}{$50 \%$} \\
\hline Trial & 1 & 2 & 3 & 1 & 2 & 3 \\
\hline$\overline{\Delta_{M}}\left(\mathrm{~ms}^{-1}\right)$ & 0.04 & 0.03 & 0.03 & -0.01 & 0.01 & 0.07 \\
$\sigma\left(\Delta_{M}\right)\left(\mathrm{ms}^{-1}\right)$ & 0.7 & 0.69 & 0.7 & 0.4 & 0.55 & 0.75 \\
Regression offset $\left(\mathrm{ms}^{-1}\right)$ & 0.027 & 0.033 & 0.02 & -0.013 & -0.043 & -0.171 \\
Regression slope & 0.987 & 0.988 & 0.991 & 1.004 & 1.005 & 1.015 \\
$r_{\text {wspd }}$ & 0.978 & 0.978 & 0.978 & 0.993 & 0.987 & 0.976 \\
$\Delta_{\alpha}($ deg $)$ & -0.78 & -0.73 & -0.86 & -1.17 & -1.07 & -0.51 \\
$\sigma\left(\Delta_{\alpha}\right)(\mathrm{deg})$ & 19.05 & 19.25 & 19.14 & 5.95 & 6.68 & 12 \\
\hline
\end{tabular}

averaged in time and then interpolated to the heights of the lidar range gates closest to the sonic anemometer heights. The temporal averaging procedure used scalar averaging for the wind speed and vector averaging for the wind direction. The center times of the averaging intervals were made to coincide with the center times of the PPI scans, and the durations of the averaging intervals were set equal to twice the PPI scan durations. We note that this method results in an undersampling (i.e., no overlap between averaging intervals) of the sonic anemometer data, since the time between PPI scans is $12 \mathrm{~min}$ and the nominal scan duration is about $40 \mathrm{~s}$.

To avoid tower wake effects, only those anemometers on the upwind side of the tower were used in the comparison with the CDL measurements. For each tower level and averaging interval, the upwind side was defined by the azimuth sector that was within $\pm 90^{\circ}$ of the mean wind direction, as determined from a vector average of the two sonic anemometers. The temporally averaged (upwind) sonic anemometer data were then interpolated to the heights of the lidar range gates closest to the sonic anemometer heights (these heights were 142.9, 194.9, 246.8 and $298.8 \mathrm{~m}$ ). The interpolation was handled by interpolating the horizontal vector components, rather the wind speeds and direction.

The CDL measurements represent a convolution (in the range dimension) of the instantaneous radial velocity with the laser pulse range weighting function (RWF) and the range gate length (Frehlich and Cornman, 2002). The size of the lidar's probe volume is defined by the width of the Gaussian laser pulse and the transverse extent of the beam, which is roughly $10 \mathrm{~cm}$ for the ARM CDL in the near-range. By contrast, the sonic anemometer may be regarded as a point measurement.

In an effort to account for the spatial averaging that is inherent in the CDL measurements we applied an estimate of the CDL's RWF to the sonic anemometer data. This was also used as a means of interpolating the sonic anemometer measurements to the height coordinates of the CDL. This inter- polation takes the following form:

$$
\gamma_{\mathrm{s}}(z)=\frac{\sum_{z_{\mathrm{s}}}^{\operatorname{RWF}\left(z_{\mathrm{s}}-z\right)} \gamma_{\mathrm{s}}\left(z_{\mathrm{s}}\right)}{\sum_{z_{\mathrm{s}}}^{\operatorname{RWF}\left(z_{\mathrm{s}}-z\right)},}
$$

where $\gamma_{\mathrm{s}}\left(z_{\mathrm{s}}\right)$ denotes either the $u$ or $v$ component of the sonic winds at the sonic anemometer height, $z_{\mathrm{s}}$, and $\gamma_{\mathrm{s}}(z)$ is the corresponding interpolated value at height $\mathrm{z}$. The range weighting function is given by (Banakh and Smalikho, 1997; Lundquist et al., 2015)

$\operatorname{RWF}(x)=\frac{1}{2 \Delta r}\left[\operatorname{erf}\left(f_{+}(x)\right)-\operatorname{erf}\left(f_{-}(x)\right)\right]$,

where

$f_{ \pm}(x)=\frac{2 \ln (2)}{\Delta p}|x / \sin \theta \pm \Delta r / 2|$,

$\Delta p$ is the laser pulse length, $\Delta r$ is the range gate length and $\theta$ is the elevation angle. The laser pulse length for the lidar used in this study is $\Delta p \sim 22.5 \mathrm{~m}$, and the range gate length was set to $30 \mathrm{~m}$. Figure 5 illustrates the range weightings for the four lidar range gates used in the comparison with the tower. It is clear that the range weighting has little effect on the sonic anemometer data because the instrument spacing is larger than the half-width of the weighting functions and the lidar range gate centers are close to the sonic heights. Thus, for this geometry, the application of Eq. (14) is essentially equivalent to using the nearest-neighbor approximation.

Once the sonic data have been temporally averaged and vertically interpolated, the comparison with the lidar is carried out by computing statistics of the wind speed difference

$\Delta_{M}=M-M_{\mathrm{s}}$,

and the wind direction difference,

$\Delta_{\alpha}=\tan ^{-1}\left(\frac{\sin \alpha \cos \alpha_{s}-\cos \alpha \sin \alpha_{s}}{\sin \alpha \sin \alpha_{s}+\cos \alpha \cos \alpha_{s}}\right)$,

where $\alpha=\tan ^{-1}\left(u_{o} / v_{o}\right)$ is the azimuth angle of the horizontal velocity vector from the lidar, $M_{\mathrm{S}}$ is the sonic anemometer wind speed and $\alpha_{s}$ is the azimuth angle of the horizontal velocity vector as determined from the sonic anemometer data. The wind direction difference $\Delta_{\alpha}$ is positive when the lidar winds are rotated clockwise relative to the sonic winds. We note that although Eq. (18) is mathematically equivalent to $\alpha-\alpha_{s}$, it is not prone to the problems that occur due to the cyclical nature of the angles.

Table 2 summarizes the results of the comparison between the CDL wind retrievals and the BAO tower for trials 1,2 and 3 . These results represent averages taken over all four heights (142.9, 194.9, 246.8 and 298.8 m) and over the deployment period from 6 March through 16 April 2015. Statistics were 


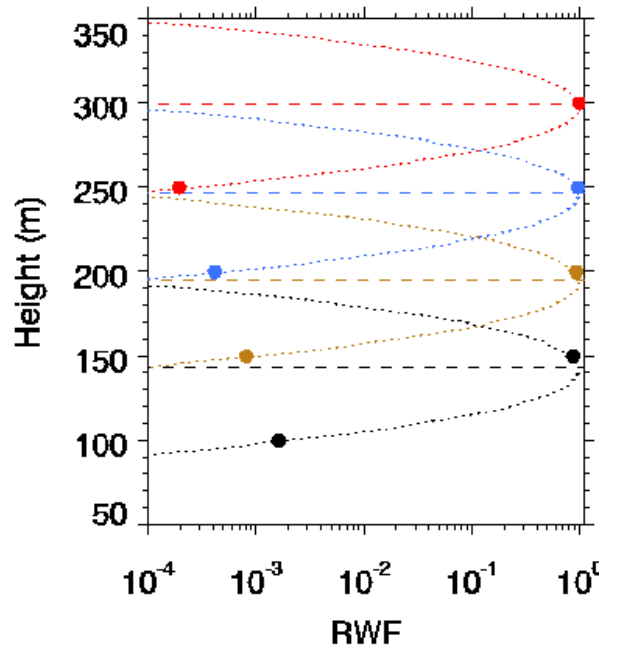

Figure 5. Range weighting functions (RWFs) used to interpolate the sonic measurements to the heights of the lidar range gates. The dashed horizontal lines show the heights of the lidar range gates, and the solid filled circles show the heights of the sonic anemometers and the range-weighting values that were used in Eq. (14). Different colors are used to distinguish the lidar range gates and corresponding RWFs.

computed by excluding lidar measurements corresponding to wind speeds less than $0.5 \mathrm{~ms}^{-1}$ in order to filter out less reliable wind direction data. The total sample count for each trial was nominally 13000 .

The wind speed bias is denoted by $\overline{\Delta_{M}}$ and the standard deviation of the wind speed difference is denoted by $\sigma\left(\Delta_{M}\right)$. Similarly, the wind direction bias is denoted by $\overline{\Delta_{\alpha}}$ and the standard deviation of the wind direction difference is denoted by $\sigma\left(\Delta_{\alpha}\right)$. Also shown is the slope and offset (i.e., intercept) of the linear regression between the sonic anemometer and CDL-derived wind speeds, and the Pearson correlation coefficient, $r_{\mathrm{wspd}}$, between the sonic and lidar wind speeds.

The results shown in Table 2 are divided into two data quality control categories. The first category uses no data rejection. In this category all of the measurements are used in the computation of the statistics, regardless of the estimated precision. The second category (i.e., last three columns of Table 2) shows the results with $50 \%$ data rejection. In this category, measurements with estimated relative wind speed precisions in the upper 50th percentile are not used in the computation of the statistics.

Table 2 shows that wind speed biases range from -1 to $7 \mathrm{~cm} \mathrm{~s}^{-1}$, and wind direction biases tend to cluster near $-1^{\circ}$. A Student's $t$ test for paired data (Press et al., 1988) suggests that these biases, albeit small, are statistically significant. Table 2 also shows that the wind speed and wind direction biases are insensitive to the treatment of the radial velocity precision in Eq. (1) and are not affected significantly by data rejection. For $0 \%$ data rejection all three trials produce similar results. The differences between the trials are more evident
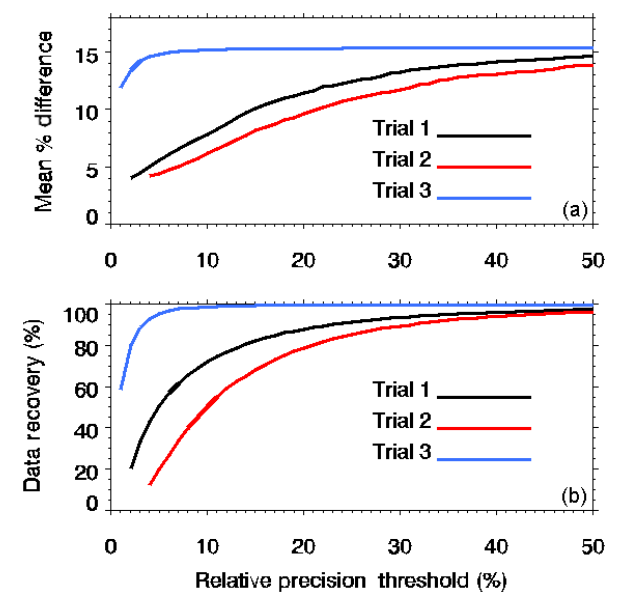

Figure 6. (a) Mean percent difference in wind speed between the CDL and the tower and (b) the data recovery as functions of the relative precision threshold. The black, red and blue curves correspond to trials 1,2 and 3 respectively.

when we compare results with and without data rejection. The results for trials 1 and 2 show significant improvement in the wind speed difference standard deviation, regression and correlation as the data rejection rate is increased from 0 to $50 \%$. By contrast, Trial 3 shows no improvement in these quantities, suggesting that the precision estimates for Trial 3 are poor indicators of data quality.

A common method of data quality control involves rejecting measurements whose estimated relative precisions, $\sigma_{M}^{\text {est }} / M$, exceed some prescribed threshold value. Figure $6 \mathrm{a}$ shows the mean percent difference in the wind speed, i.e., $100 \times \overline{\left|M-M_{\mathrm{S}}\right| / M_{\mathrm{S}}}$, as a function of the estimated relative precision threshold. As this threshold is increased for a given trial, the mean percent wind speed difference asymptotically approaches a limiting value. Trial 3 converges to its limiting value much more quickly than trials 2 or 3 . For a given threshold, trials 2 and 3 produced the smallest and largest percent differences, respectively.

The application of the relative precision threshold reduces the sample population, or data recovery. Figure $6 \mathrm{~b}$ shows data recovery for trials 1,2 and 3 as a function of the relative precision threshold. The data recovery is the percentage of measurements with estimated relative precisions below the prescribed threshold. For a given threshold level trials 2 and 3 give the lowest and highest data recoveries, respectively. Trial 3 produces high data recoveries because the estimated precisions are generally much lower than either Trial 1 or 2. Thus, for a given threshold level fewer measurements are rejected for Trial 3.

To assess the absolute accuracy of the precision estimates, Fig. 7a shows the relationship between the mean absolute wind speed difference and the estimated wind speed precision, $\sigma_{M}$. Similarly, Fig. $7 \mathrm{~b}$ shows the mean absolute wind direction difference as a function of the estimated wind di- 

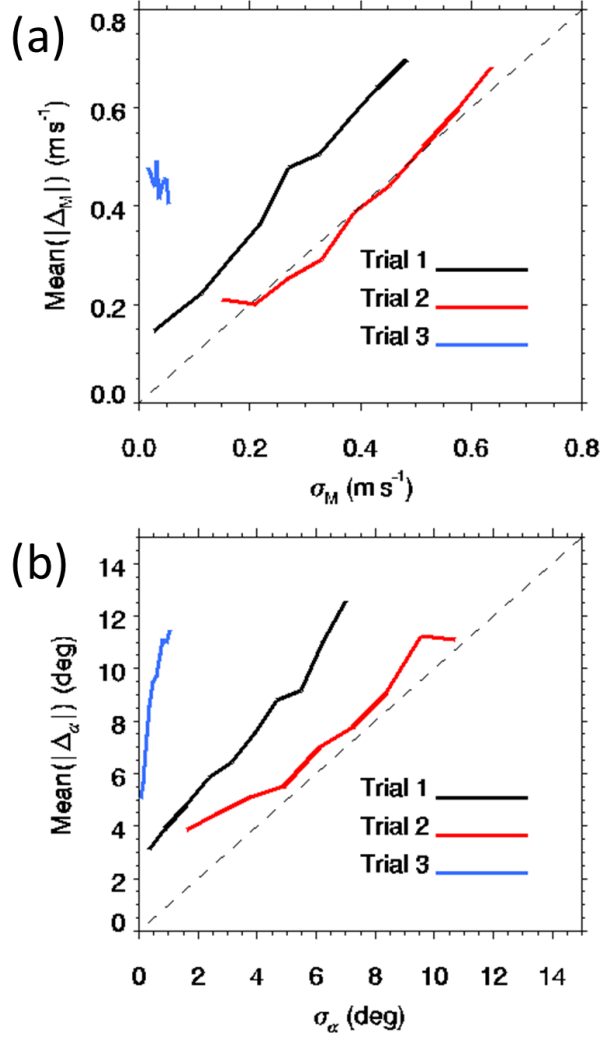

Figure 7. (a) Mean absolute difference in wind speed between the $\mathrm{CDL}$ and the BAO tower sonic anemometers as a function of the estimated wind speed precision, $\sigma_{M}$. (b) Mean absolute difference in wind direction between the CDL and tower as a function of the estimated wind direction precision, $\sigma_{\alpha}$. The black, red and blue curves show the results for trials 1,2 and 3 , respectively.

rection precision, $\sigma_{\alpha}$. These curves were obtained by sorting the estimated precisions into discrete bins and computing the absolute wind speed and direction differences within each bin. These results show that Trial 2 produced precision estimates that agree well with the observed differences, and Trial 1 produces estimates that are about $30 \%$ smaller. By contrast, Trial 3 produces estimates that are far smaller than the observed differences and compressed into a relatively narrow range of values.

\subsection{Diurnal variability}

As seen in Fig. 4, the estimated wind speed precisions for trials 1 and 2 both show similar diurnal variations. To investigate this dependence further, Fig. 8a shows the mean absolute difference in wind speed and mean absolute wind direction difference between the CDL and the tower as functions of the time of day for Trial 2. These results were obtained by averaging over the deployment period from 6 March through 16 April 2015. The lidar wind retrievals were quality controlled by rejecting measurements with estimated relative wind speed precisions greater than $25 \%$. Also, Fig. $8 \mathrm{~b}$ shows (a)
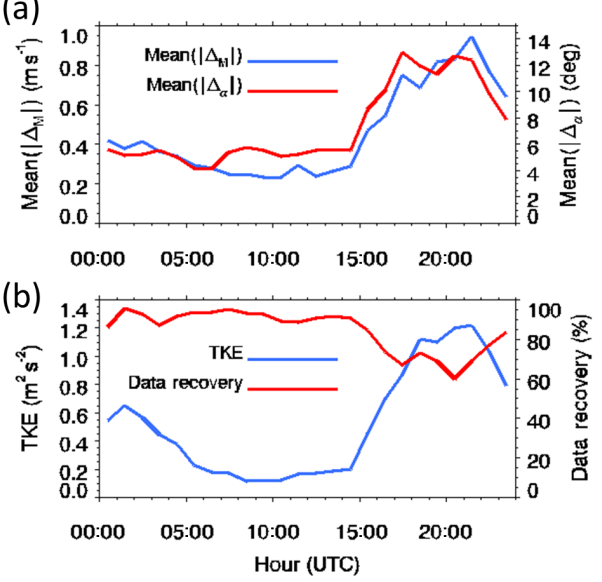

Figure 8. (a) Diurnal variation of the mean absolute wind speed difference (blue) and the mean absolute wind direction difference (red) between the CDL and the tower sonic anemometers for Trial 2. (b) Diurnal variation of the TKE (blue) and the data recovery (red). The CDL wind retrievals were quality controlled using a relative estimated precision threshold of $25 \%$.

the turbulent kinetic energy (TKE) and the CDL data recovery as functions of the time of day. The TKE was computed from the sonic anemometer data by taking the median value of 30-minute-averaged velocity variances over four levels on the tower $(150,200,250$ and $300 \mathrm{~m})$. We note that sunrise and sunset occurred at approximately 12:00 and 02:00 UTC, respectively.

Figure 8 shows that the mean absolute wind speed difference between the CDL and tower sonic anemometers is nominally around $30 \mathrm{~cm} \mathrm{~s}^{-1}$ at night and then increases to about $90 \mathrm{~cm} \mathrm{~s}^{-1}$ near solar noon. Similarly, the mean absolute wind direction difference is roughly $5^{\circ}$ at night and then increases to about $12^{\circ}$ near solar noon. The observed wind speed and wind direction differences increase as turbulence levels increase. Thus, the diurnal variation in the wind speed and wind direction differences is strongly correlated with the diurnal variation in TKE (Fig. 8b). The data recovery is approximately anticorrelated with the TKE. The data recovery reaches a maximum of just over $90 \%$ at night and a minimum of between 60 and $70 \%$ during the day.

\section{Summary}

This study evaluated the accuracy of wind speed and wind direction precision estimates as computed from CDL scan data using standard error propagation techniques in a VAD algorithm. Precision estimates were compared to differences in wind speed and direction between the VAD algorithm and the sonic anemometers mounted on the $300 \mathrm{~m} \mathrm{BAO}$ tower. Three wind retrieval trials were conducted using different schemes for estimating the precision in the retrieved wind speed and direction. Comparisons were carried out using data from the 
XPIA field campaign in which one of the ARM CDLs was deployed in close proximity to the BAO tower.

For Trial 1 the radial velocity precisions were assumed to be unknown, so that the precisions in the retrieved velocity components were estimated from the diagonal elements of the unweighted covariance matrix and the fit residual, ie. Eq. (7). This approach is equivalent to assuming the radial velocity uncertainty to be isotropic. Trial 2 was a proxy for a PPI sampling strategy in which multiple profiles are acquired for each pointing direction. This method allows for direct computation of the radial velocity variance and avoids the assumption of isotropy inherent in Trial 1. For Trial 3 the effects of turbulence were completely ignored, and the radial velocity precision was set equal to the instrumental measurement precision. Since the measurement precision is parameterized in terms of the SNR, the radial velocity precision for Trial 3 was determined solely by the SNR.

Our results showed that all trials produced qualitatively similar wind fields, with negligible bias when compared to the BAO tower sonic anemometers. There were, however, substantial differences in the precision estimates between the three trials. The precisions for trials 1 and 2 were well correlated but different in magnitude, with Trial 1 producing estimates that were about $30 \%$ smaller than Trial 2. Both trials 1 and 2 exhibited a similar diurnal variation, with larger precisions occurring during the daytime under convective conditions. By contrast, the precision estimates for Trial 3 were much smaller in magnitude and poorly correlated with either trials 1 or 2 .

Trial 2 resulted in the best agreement between the wind speed and direction precision estimates from the VAD algorithm and the mean absolute difference in wind speed and direction between the VAD algorithm and the tower. Filtering the retrieved winds based on the relative estimated wind speed precision resulted in a substantial improvement in the agreement between the retrieved winds and the tower for trials 1 and 2 and little improvement for Trial 3. This behavior suggests that the precision estimates from Trial 3 are poor indicators of data quality.

An important lesson here is that it is inappropriate to equate the radial velocity precision, $\sigma_{\mathrm{r}}$, to the instrumental measurement precision, $\sigma_{\mathrm{n}}$, in the wind retrieval algorithm. In the context of the VAD algorithm, turbulent fluctuations are a source of error, so ignoring this error results in wind speed and direction precision estimates that are biased low. Best results are achieved when $\sigma_{\mathrm{r}}$ can be determined directly from the standard deviation of the radial velocity along each pointing direction and range gate of the PPI scan.

The precision estimation scheme of Trial 1 represents the current method that is used operationally by the ARM program for computing wind profiles from the all ARM CDLs (these data are publicly available through the ARM website at http://www.arm.gov/). This method is simple to implement, produces reasonable results and requires only a standard "single-pass" PPI scan to run. Another important advan- tage is that the single-pass PPI scan can be executed quickly, thereby minimizing interruptions to vertical velocity profiling, which is a high scientific priority among ARM data users.

Further testing is required in order to evaluate scanning strategies that enable direct computation of the radial velocity variance (as in Trial 2) while minimizing the impact on the vertical velocity profiling. Practical implementation could be achieved using a contiguous sequence of PPI scans with acquisition of a single profile in each pointing direction or by acquiring multiple profiles along a given pointing direction before moving on to the next pointing direction. The latter method is more efficient since it minimizes the downtime associated with moving the scanner from one position to the next, thereby minimizing the total scan time. Additionally, there are other tradeoffs to be considered such as reducing the pulse integration time and perhaps reducing the number of pointing directions (while retaining at least four or more pointing directions), with the goal of keeping the total scan time to about $1 \mathrm{~min}$ or less.

Data availability. Raw data used in this study are available through the "Atmosphere to Electrons" (A2e) website at https://a2e.energy. gov/. Access is not restricted, but new users must first register in order to obtain a username and password. From the main A2e web page, select "Data" and then select "XPIA". The BAO high-frequency sonic anemometer data can be accessed by selecting "ecor.z01.00". The tilt-corrected sonic anemometer data used in this study are stored in "ecor.z01.tar". The process for obtaining the raw PPI scan data from the lidar is similar. From the main A2e web page, select "Data" and then select "XPIA". The raw PPI scan data from the ARM Doppler lidar can be accessed by selecting "lidar.z06.00". The data for each PPI scan are stored in separate netCDF files with the following file naming convention: "lidar.z06.YYYYMMDD.hhmmss.vad_b1.nc", where "YYYYMMSS" is the year, month, and day, and "hhmmss" is the hour, minute, and second of the start of the PPI scan.

Competing interests. The authors declare that they have no conflict of interest.

Acknowledgements. This research was supported by the Office of Biological and Environmental Research of the US Department of Energy as part of the Atmospheric Radiation Measurement Climate Research Facility. Funding for XPIA was provided by the US Department of Energy, Office of Energy Efficiency and Renewable Energy, Wind and Water Power Technologies Office, and by NOAA's Earth System Research Laboratory. We express our appreciation to the National Science Foundation's CABL program (https://www.eol.ucar.edu/field_projects/cabl) for supporting the deployment and operation of the sonic anemometers on the BAO tower. We also thank Scott Sandberg, Aditya Choukulkar and Paul Quelet for their assistance with the deployment of the ARM Doppler lidar. 
Edited by: A. Clifton

Reviewed by: two anonymous referees

\section{References}

Banakh, V. A. and Smalikho, I. N.: Estimation of turbulent energy dissipation rate from data of pulse Doppler lidar, Atmos. Ocean. Opt., 10, 957-965, 1997.

Browning, K. and Wexler, R.: The determination of kinematic properties of a wind field using Doppler radar, J. Appl. Meteorol., 7, 105-113, 1968.

Chai, T., Lin, C. L., and Newsom R. K.: Retrieval of Microscale Flow Structures from High Resolution Doppler Lidar using an Adjoint Model, J. Atmos. Sci., 61, 1500-1520, 2004.

Choukulkar, A., Pichugina, Y., Clack, C. T. M., Calhoun, R., Banta, R., Brewer, A., and Hardesty, M.: A new formulation for rotor equivalent wind speed for wind resource assessment and wind power forecasting, Wind Energy, 19, 1439-1452, 2016.

Clifton, A., Elliot, D., and Courtney, M.: IEA Wind RP 15 Ground-based vertically-profiling remote sensing for wind resource assessment, International Energy Agency, available at: https://www.ieawind.org/index_page_postings/RP/RP2015_ RemoteSensing_1stEd_8March2013.pdf (last access: 27 March 2017), 2013.

Davies, F., Collier, C. G., Bozier, K. E., and Pearson, G. N.: On the accuracy of retrieved wind information from Doppler lidar observations, Q. J. Roy. Meteorol. Soc., 129, 321-334, 2003.

Frehlich, R: Velocity error for coherent Doppler lidar with pulse accumulation, J. Atmos. Ocean. Tech., 21, 905-920, 2004.

Frehlich, R. and Cornman, L.: Estimating Spatial Velocity Statistics with Coherent Doppler Lidar, J. Atmos. Ocean. Tech., 19, 355366, 2002.

Gottschall, J.,Courtney, M. S., Wagner, R., Jorgensen, H. E., and Antoniou, I.: Lidar profilers in the context of wind energy: a verification procedure for traceable measurements, Wind Energy, 15, 147-159, 2012.

Hsuan, C. Y., Tasi, Y. S., Ke, J. H., Prahmana, R. A., Chen, K. J., and Lin, T. H.: Validation and Measurements of Floating LiDAR for Nearshore Wind Resource Assessment Application, The 6th International Conference on Applied Energy - ICAE2014, Energy Procedia, 61, 1699-1702, 2014.

Kaimal, J. C. and Gaynor, J. E.: The Boulder Atmospheric Observatory, J. Clim. Appl. Meteorol., 22, 863-880, 1983.

Koch, G. J., Beyon, J. Y., Modlin, E. A., Petzar, P. J., Woll, S., Petros, M., Yu, J., and Kavaya, M. J.: Side-scan Doppler Lidar for Offshore Wind Energy Applications, J. Appl. Remote Sens., 6, 063562-1-063562-11, doi:10.1117/1.JRS.6.063562, 2012.

Lane, S. E., Barlow, J. F., and Wood, C. R.: An assessment of a three-beam Doppler lidar wind profiling method for use in urban areas, J. Wind. Eng. Ind. Aerod., 119, 53-59, 2013.

Lang, S. and McKeogh, E.: LIDAR and SODAR Measurements of Wind Speed and Direction in Upland Terrain for Wind Energy Purposes, Remote Sens., 3, 1871-1901, doi:10.3390/rs3091871, 2011

Lenschow, D. H., Wulfmeyer, V., and Senff, C.: Measuring Secondthrough Fourth-Order Moments in Noisy Data, J. Atmos. Ocean. Tech., 17, 1330-1347, 2000.
Lundquist, J. K., Churchfield, M. J., Lee, S., and Clifton, A.: Quantifying error of lidar and sodar Doppler beam swinging measurements of wind turbine wakes using computational fluid dynamics, Atmos. Meas. Tech., 8, 907-920, doi:10.5194/amt-8-9072015, 2015.

Lundquist, J. K., Wilczak, J. M., Ashton, R., Bianco, L., Brewer, W. A., Choukulkar, A., Cliftn Mithu Debnath, A., Friedrich, R. D. K., Gunter, S., Hamidi, A., Iungo, G. V., Kaushik, A., Langan, B. K. P., Lavin, A. L. E., Lee, J. C.-Y., McCaffrey, K. L., Newsom, R. K., Noone, D. C., Oncley, S. P., Quelet, P. T., Sandberg, S. P., Schroeder, J. L., Shaw, W. J., Sparling, L., St. Martin, C., St. Pe, A., Strobach, E., Tay, K., Vanderwende, B., Weickmann, A., Wolfe, D., and Worsnop, R.: Assessing state-ofthe-art capabilities for probing the atmospheric boundary layer: the XPIA field campaign, B. Am. Meteorol. Soc., 98, 289-314, doi:10.1175/BAMS-D-15-00151.1, 2016.

Mather, J. H. and Voyles, J. W.: The ARM Climate Research Facility: A Review of Structure and Capabilities, B. Am. Meteorol. Soc., 94, 377-392, 2013.

McCaffrey, K., Quelet, P. T., Choukulkar, A., Wilczak, J. M., Wolfe, D. E., Oncley, S. P., Brewer, W. A., Debnath, M., Ashton, R., Iungo, G. V., and Lundquist, J. K.: Identification of tower-wake distortions using sonic anemometer and lidar measurements, Atmos. Meas. Tech., 10, 393-407, doi:10.5194/amt-10-393-2017, 2017.

Newman, J. F., Bonin, T. A., Klein, P. M., Wharton, S., and Newsom, R. K.: Testing and validation of multi-lidar scanning strategies for wind energy applications, Wind Energy, doi:10.1002/we.1978, 2016.

Newsom, R. K.: Doppler Lidar Handbook, DOE/SC-ARM-TR-101, 2012.

Newsom, R. K. and Banta, R. M.: Assimilating Coherent Doppler Lidar Data into a Model of the Atmospheric Boundary Layer: Part I, Algorithm Development, J. Atmos. Ocean. Tech., 21, 1328-1345, 2004a.

Newsom, R. K. and Banta, R. M.: Assimilating Coherent Doppler Lidar Data into a Model of the Atmospheric Boundary Layer: Part II, Sensitivity Analyses, J. Atmos. Ocean. Tech., 21, 1809_ 1824, 2004b.

Newsom, R. K., Ligon, D., Calhoun, R., Heap, R., Cregan, E., and Princevac, M.: Retrieval of microscale wind and temperature fields from single- and dual-Doppler lidar data, J. Appl. Meteorol., 44, 1324-1345, 2005.

Newsom, R. K., Berg, L. K., Shaw,W. J., and Fischer, M. L. Turbine-scale wind field measurements using dual-Doppler lidar, Wind Energy, 18, 219-235, doi:10.1002/we.1691, 2015.

Pearson, G., Davies, F., and Collier, C.: An Analysis of the Performance of the UFAM Pulsed Doppler Lidar for Observing the Boundary Layer, J. Atmos. Ocean. Techn., 26, 240-250, 2009.

Pena, A., Hasager, C. B., Gryning, S. E., Courtney, M., Antoniou, I., and Mikkelsen, T.: Offshore Wind Profiling using Light Detection and Ranging Measurements, Wind Energy, 12, 105-124, 2009.

Pichugina, Y. L., Banta, R. M., Brewer, W. A., Sandberg, S. P., and Hardesty, R. M.: Doppler Lidar-Based Wind-Profile Measurement System for Offshore Wind Energy and Other Marine Boundary Layer Applications, J. Appl. Meteorol. Climatol., 51, 327-349, 2012. 
Press, W. H., Flannery, B. P., Teukolsky, S. A., and Vetterling, W. T.: Numerical Recipies in C, Cambridge University Press, Cambridge, 526-534, 1988.

Pu, Z., Zhang, L., and Emmitt, G. D.: Impact of airborne Doppler wind lidar profiles on numerical simulations of a tropical cyclone, Geophys. Res. Lett., 37, L05801, doi:10.1029/2009GL041765, 2010.

Riishøjgaard, L. P., Atlas, R., and Emmitt, G. D.: The Impact of Doppler Lidar Wind Observations on a Single-Level Meteorological Analysis, J. Appl. Meterol., 43, 810-820, 2004.
Wang, H., Barthelmie, R., Clifton, A., and Pryor, S.: Wind Measurements from Arc Scans with Doppler Wind Lidar, J. Atmos. Ocean. Tech., 32, 2024-2040, doi:10.1175/JTECH-D-14$00059.1,2015$.

Wilczak, J. M., Oncley, S. P., and Stage, S. A.: Sonic anemometer tilt correction algorithms, Bound.-Lay. Meteorol., 99, 127-150, 2001.

Weissmann, M. and Cardinali, C.: Impact of airborne Doppler lidar observations on ECMWF forecasts, Q. J. Roy. Meteorol. Soc., 133, 107-116, doi:10.1002/qj.16, 2007. 